\title{
20
}

\section{Partial-Frame Retransmission Scheme for Data Communication Error Recovery in B-ISDN}

\author{
Ichiro INOUE and Naotaka MORITA
}

Communication Switching Laboratories, Nippon Telegraph and Telephone Corporation, 3-9-11 Midori-cho, Musashino-shi, Tokyo 180, Japan

\begin{abstract}
An increasing number of computers and workstations will require data communications like that of LAN-LAN interconnections. The requirement for high end-to-end throughput will be satisfied by B-ISDN, in which the ATM technique is the key technology. In B-ISDN, user data is segmented into fixed-length short packets (cells) and transferred at very high speed. The greater the amount of user data to be transmitted at one time, the more efficient the transmission has to be. Efficient error recovery is needed for the higher transmission efficiency as well as for a shorter response time, which is also a key data communication requirement.

This paper describes the functions needed for data communication in B-ISDN and classifies the error recovery methods that can be used by the end-to-end protocol for data communication. A partial-frame retransmission scheme is proposed and its performance is compared to that for selective-frame retransmission from the viewpoints of implementation complexity, efficiency, total throughput, and cell-loss probability.
\end{abstract}

A functional block model for partial-frame retransmission is proposed and it is shown that partial-frame retransmission can be implemented with little addition to the model for selective-frame retransmission.

The evaluation results show that partial-frame retransmission can provide higher efficiency for a wide variety of parameter values compared with selective-frame retransmission. They also show that partial frame retransmission reduces the cell-loss rate to less than $10 \%$ of that for selective-frame retransmission. In particular, when the incoming traffic load is relatively high, the proposed partialframe retransmission scheme can avoid the congestion that selective-frame retransmission allows.

Keyword Codes: C.2.2; C.2.0; C.2.1

Keywords: Network Protocols; Computer-Communication Networks, General; Network Architecture and Design 


\section{Introduction}

With the continuing speed of processing capability in personal computers, workstations, and servers, conversational data communication between computers and distributed processing among processors increases the requirements for inter-computer communication. The expansion of the area in which computers communicate with each other strengthens the requirements for inter-local-area data communication, like LAN-LAN interconnections, as well for wide-area data communication, like wide-area networks (WANs). Applications for such inter-computer communication include the traditional LAN applications (file transfer, remote procedure call, transaction processing, and LAN management message exchange), the WAN signaling message exchange, and the large file transfer for WAN management and operation, e.g., Telecommunications Management Network (TMN[1]).

Data communication traffic is generally very bursty, with a peak rate as high as several Mbps. Typical traffic in a LAN or LAN-LAN-interconnect environment is the exchange of control data units for managing and maintaining the network configuration. Signaling traffic is the exchange of the relatively short messages that occurs for call control (set up, release, etc.). The TMN traffic includes both large file exchange and short operational message exchange. The required average rate for this kind of traffic may be relatively low since a large file transfer with a high peak rate does not often occur. On the other hand, when a typical application in such an environment, e.g., file transfer, occurs, the peak rate can be as high as the physical medium's speed.

Data communication is currently handled with leased lines, X.25 services, or dedicated networks (in the case of No. 7 signaling). None of these alternatives, however, meets the requirement for high speed (bit rate) with efficient transmission (cost).

B-ISDN will provide multimedia communication services for voice, images, motion pictures, data, etc. Asynchronous transfer mode (ATM[2][3]), which is based on the asynchronous transfer of fixed-size packets (cells) provides a wide range of communication rates, as well as variable-rate communication, while providing statistical multiplexing capability. Therefore, ATM is expected to be the common transfer technique in B-ISDN. ATM-based B-ISDN will provide both high-bit-rate, up to hundreds of Mbps, and efficient transmission.

B-ISDN will initially provide connection-oriented services suitable, for example, for LAN-LAN interconnections. In LAN-LAN interconnections there can be many types of data communication applications, such as file transfer, transaction processing, image referencing, electronic mailing, and computer-aided design. However, many problems remain to be solved before such applications can economically use B-ISDN's high performance.

One problem to be solved is the protocol for data communication. Voice, motion picture, image, and data communication services require different transfer characteristics, but ATM provides only uniform transfer characteristics. Thus, an ATM adaptation layer (AAL[4][5]) protocol is needed to adapt the ATM layer capability to provide service-specific characteristics. The AAL protocol for data communication should provide flow control for high-speed data transmission, error 
control for error correction, and congestion control to avoid network congestion and to prevent user buffer overflow.

This paper clarifies the requirements for the AAL protocol for high-speed data communication in B-ISDN in Section 2. Section 3 shows examples of error recovery methods and proposes a partial-frame retransmission method for error recovery; a block diagram shows that the proposed method can be implemented easily. Section 4 gives the theoretical and simulated evaluation results for the current selective-frame retransmission method and the proposed partial-frame retransmission method. The results show that the proposed partial-frame retransmission method meets the requirements for data communication. Section 5 summarizes the paper and mentions remaining problems.

\section{Data communication in B-ISDN}

A typical point-to-point B-ISDN data communication model (Fig. 1) has two end terminals that communicate by segmenting and reassembling the data units (frames) into and from the ATM cells, which have a fixed 53-byte length.

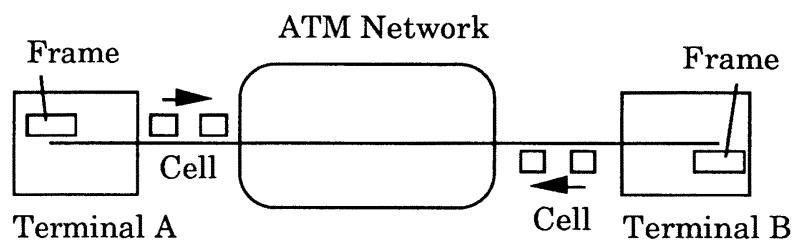

Figure 1. B-ISDN data communication model.

The B-ISDN environment can be characterized by its high-speed linking over virtual channels (VCs), by its low to moderate cell-loss rate depending on the required multiplexing effect, and by its small to large round-trip delay depending on the distance between the source and destination entities. The data communication requirements in B-ISDN are high speed, a low end-to-end error rate, high efficiency, and a quick response time. To meet these requirements, a simple functional configuration, proper flow control, and proper error control are needed. Efficient error recovery is especially needed to achieve low overhead and reduce traffic volume.

Two possible protocol stacks for B-ISDN data communication are shown in Fig. 2. Combination (1) has a service-specific convergence sublayer (SSCS), which may include a service-specific coordination function (SSCF) and a service-specific connection-oriented protocol (SSCOP[6]) over an AAL-common part (CP) (types $3 / 4$ or 5 ) over the ATM and physical (PHY) layers as shown in Fig.3. Combination (2) uses the existing protocol over RFC 1483[7] over the AAL-CP5, ATM, and PHY layers. In both cases, the upper layer relies on the underlying transmission provided by the AAL, ATM, and PHY layers.

Figure 3 shows the data communication protocol structure recently standardized by the ITU-T. The figure focuses on the internal structure of AAL Types $3 / 4$ and 
5. In addition to the functions shown for each sublayer, the SSCS provides end-toend frame error recovery if necessary.

\begin{tabular}{|c|}
\hline Upper Layer \\
\hline $\begin{array}{c}\text { SSCS } \\
\text { (SSCF/SSCOP) }\end{array}$ \\
\hline $\begin{array}{c}\text { AAL-CP } \\
\text { (Type } 3 / 4 \text { or } 5)\end{array}$ \\
\hline ATM \\
\hline PHY \\
\hline
\end{tabular}

(1) Combination using SSCS

\begin{tabular}{|c|}
\hline $\begin{array}{c}\text { Upper Layer } \\
\text { (Existing Protocol) }\end{array}$ \\
\hline RFC 1483 \\
\hline AAL-CP Type 5 \\
\hline ATM \\
\hline PHY \\
\hline
\end{tabular}

SSCS: Service Specific Convergence Sublayer

SSCF: Service Specific Coordination Function SSCOP: Service Specific Connection Oriented Protocol

\section{AAL: ATM Adaptation} Layer

2) Combination using CP: Common Part RFC1483

Figure 2. Possible protocol stacks for B-ISDN data communication.

\begin{tabular}{|c|c|c|c|c|}
\hline \multicolumn{4}{|c|}{ Upper Layers } & Example functions \\
\hline \multirow{4}{*}{ A.A. } & \multirow{3}{*}{ es } & \multirow{2}{*}{ SSCS } & SSCF & Primitive mapping, retrieval invocation for signalling \\
\hline & & & SSCOP & Error control, flow control, link reset, keep alive, retrieval \\
\hline & & \multicolumn{2}{|c|}{ CPCS } & CS-PDU validation, buffer allocation (3/4), CRC (5) \\
\hline & \multicolumn{3}{|c|}{ SAR } & Cell<->AAL PDU mapping, CRC (3/4), multiplexing (3/4) \\
\hline \multicolumn{4}{|c|}{ ATM Layer } & Cell transfer, header error check, loss priority control \\
\hline \multicolumn{4}{|c|}{ Physical Layer } & Bit transfer, synchronous multiplexing (SDH) \\
\hline
\end{tabular}

SSCF: Service-Specific Coordination Function ATM: Asynchronous Transfer Mode SSCOP: Service-Specific Connection-Oriented SDH: Synchronous Digital Hierarchy Protocol

SSCS: Service-Specific CS

CPCS: Common Part CS

CS: Convergence Sublayer

CRC: Cyclic redundancy check code and associated actions

SAR: Segmentation and Reassembly Sublayer (5): Function specific to Type 5

AAL: ATM Adaptation Layer

Note: The SSCF and SSCOP sublayers are only defined for the AAL type 5 at present.

Figure 3. Framework of AAL Types $3 / 4$ and 5 for data communication.

The ATM layer provides cell-level transfer and cell-header error detection. The AAL-CP is the AAL-CPCS and AAL-SAR. It provides segmentation and 
reassembly of the AAL-CP service data unit (SDU) into and from the cell. It also provides cell-level error detection; the detection method varies for each AAL type. AAL Type 3/4 uses the cyclic redundancy check code (CRC), the cell sequence number, the message identifier, the length indicator, and the frame length. AAL Type 5 uses both the CRC and the length indicator at the frame level. The AAL service-specific part provides frame-level error detection and recovery, as well as frame-level flow control. Existing protocols, including TCP[8]/IP[9], XTP[10], VMTP[11], and high-speed data link control procedure (HDLC[12]), provide framelevel flow control and error recovery.

If RFC 1483 is used rather than SSCOP, frame-level error recovery can only be provided by the existing protocol above the RFC. If additional error recovery functions are needed, the existing protocol or RFC 1483 has to be amended to provide them.

Errors in B-ISDN may be classified into those at the bit level, those at the cell level, and those at the frame level. Bit-level errors, which may be caused by link bit errors, are detected by ATM or AAL-CP error detection. Cell-level errors are defined as cell loss due to buffer overflow or cell misinsertion. These errors can be detected by AAL-CP as well as by the AAL service-specific part (AAL-SS). They can also be detected as frame corruption or frame loss by the AAL-CP and the AAL-SS. If the bit error and cell-loss rate are not too high, even if an error occurs, the corruption may be restricted to within a part of the corrupted frame.

Although ATM and AAL-CP provide error detection, end-to-end error correction is needed for data communication because ATM cell loss cannot be recovered with only error detection. While B-ISDN generally provides a very low cell-loss rate, about $10^{-9}$, if the multiplexing gain is large, the utilized cell-loss rate may temporarily be higher[13]. In such a case, an efficient error recovery scheme is needed that minimizes the total amount of data to be transmitted and retransmitted and the probability of congestion.

\section{Error recovery schemes}

\subsection{Preventive recovery (Forward Error Control: FEC)}

\subsubsection{CRC}

\section{(a) Overview}

The cyclic redundancy check code (CRC) detects a variety of bit errors but corrects only same of them: it is targeted at bit-level error control. In the sense that no retransmission is not required for error recovery, the CRC can be defined as a kind of preventive error recovery method. The CRC is generated by processing the data according to a specific formula. When the data is received, this same formula is applied to the data and the result is compared with the CRC value contained in the received data unit.

\section{(b) Hardware implementation}

Since the CRC only uses simple addition with modulus 2 and a fixed-length delay, it is easily implemented. The processing speed can be high enough so that processing time can generally be neglected compared with the end-to-end round-trip delay. 


\section{(c) Possible Problem}

Even if the CRC is used, the data communication user may still require further error recovery by retransmission. One reason for this is that the CRC's error correction capability is limited. It can only correct a limited variety of errors (a limited number of the errored bits). Loss of data, which typically occurs due to cell loss, cannot be recovered by the CRC. Another reason is that the efficiency degrades: the CRC has to be lengthened in order to correct a greater variety of errors. Each data item needs a CRC, which leads to low efficiency, especially when the error rate is not very high.

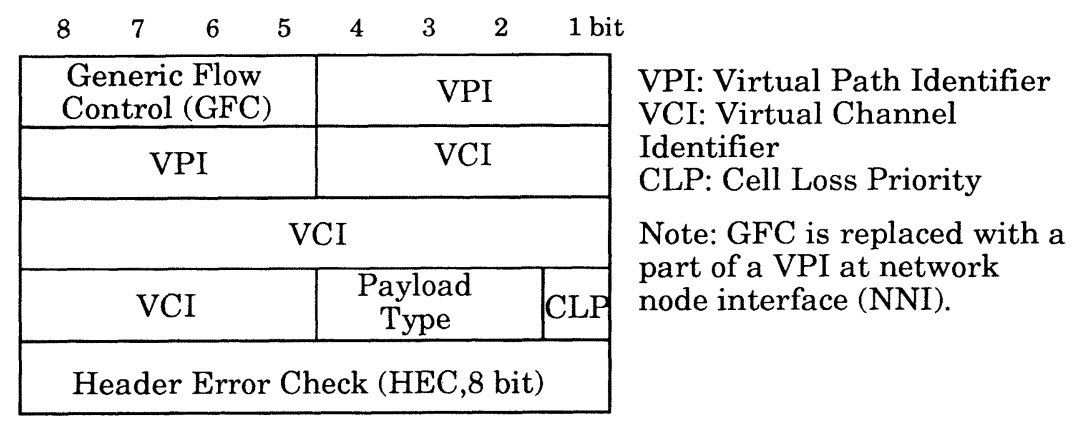

(a) Header structure of cell at UNI

\begin{tabular}{|c|c|c|c|c|c|}
\hline \multicolumn{2}{c}{4} & \multicolumn{2}{c}{10} & 6 & 10 bits \\
\hline $\begin{array}{c}\text { Segment } \\
\text { Type }\end{array}$ & $\begin{array}{c}\text { Sequence } \\
\text { Number }\end{array}$ & $\begin{array}{c}\text { Message } \\
\text { Identifier }\end{array}$ & SAR-Payload & $\begin{array}{c}\text { Length } \\
\text { Indicator }\end{array}$ & CRC \\
\hline
\end{tabular}

(b) SAR-PDU format for AAL Type 3/4

\begin{tabular}{|c|c|c|c|c|c|}
\hline \multicolumn{2}{c}{$0-47$} & 1 & 1 & 2 & 4 Bytes \\
\hline CPCS-PDU Payload & PAD & CPCS-UU & $\begin{array}{c}\text { Common } \\
\text { Part ID }\end{array}$ & Length & CRC \\
\hline
\end{tabular}

CPCS: Common Part Convergence Sublayer; :PAD: Padding;

UU: User-User data; ID: identifier

(c) CPCS-PDU format for AAL Type 5

Figure 4. CRC used in ATM and AAL layers.

\section{(d) Application}

Although CRC error detection can be easily implemented without any delay due to retransmission, further retransmission is needed for error recovery. These 
characteristics make the CRC more suitable for the lower layers, for example, ATM header error check (HEC) and the SAR CRC, as well as AAL5-CP CRC32, each of which assumes higher layer retransmission if necessary. Figure 4 shows how these CRCs are used by these protocols. The CRC may therefore not be suitable as the end-to-end error recovery method in a B-ISDN environment.

\subsubsection{PCR}

\section{(a) Overview}

Preventive cyclic retransmission (PCR[14]) autonomously retransmits the previously sent data when no new data is to be sent or the amount of already sent data reaches the preestablished default value. Therefore, if the received data is errored, the receiver can expect to receive the retransmitted data soon after receiving the original data. Using PCR to recover data errors avoids the round-trip delay that comes with the ordinary retransmission mechanism in which the receiver must request retransmission of the errored data. It is thus effective in environments with a large round-trip delay or a relatively high error rate, such as satellite links.

\section{(b) Hardware implementation}

Since PCR simply retransmits the previously sent data, implementation is not complex. It only requires that the sender and receiver have a relatively large buffer for the retransmission and the reordering, respectively.

\section{(c) Problem}

Though PCR recovers errors, including any data loss, without much delay, data is retransmitted even when it is unnecessary, which increases costs because it uses more bandwidth.

\section{(d) Application}

Since PCR provides quick recovery, but at low efficiency, it is good for leased-line users or for internal network information transfer, which are not sensitive to efficiency. It is also good when there is a very large round-trip delay, as with a satellite line. It is not suitable as the error recovery method for B-ISDN.

\subsection{Retransmission Recovery (Automatic Repeat Request: ARQ)}

Since preventive error recovery methods do not recover lost data or handle some kinds of errors, reactive recovery, i.e., retransmitting necessary information, is needed. Recovery by retransmission can be classified according to the unit of retransmission.

\subsubsection{Go-back-N frames retransmission}

This retransmission scheme retransmits all the data after an error has occurred. Therefore, it is effective in networks with moderate to low error rates, relatively small round-trip delay, and moderate to low speed. The traditional data link 
protocol, HDLC, uses this Go-back-N (GBN) scheme. HDLC is the basis for existing data link protocols, including the packet network datalink layer (X.25 LAPB[15]), the ISDN D-channel (LAPD[16]), and the frame relay control part (Q.922[17] control, LAPF). All of these protocols are based on GBN retransmission. The GBN error recovery scheme is also used by both TCP and XTP. In these two protocols, data is identified by both its length (bytes) and its segment sequence number.

\section{(a) Procedure}

In GBN retransmission, a sequence number is maintained in both the sending and receiving entities. This sequence number can be based on either the number of the data unit (frame) or the amount of data (bytes). The sender holds a copy of the sent data in its buffer until the acknowledgment for the data is received. When an error is detected, the sender retransmits all data units with sequence numbers larger than that of the errored unit. The retransmission may be invoked by a notification sent by the receiving side, which checks the continuity of the sequence numbers. This notification may also be sent by a control data unit (S-frame in HDLC, STAT PDU in SSCOP).

Figure 5 shows a sequence chart for GBN error recovery as used in HDLC. The information frame with a sequence number of 0 is lost and the next frame is received. The receiver detects the sequence number gap and requests retransmission by sending a reject frame (REJ). The received information frames are discarded before the error is recovered. In some protocols, like TCP, the sending entity also retransmits the data if the timer for the sent data expires before an acknowledgment is received.

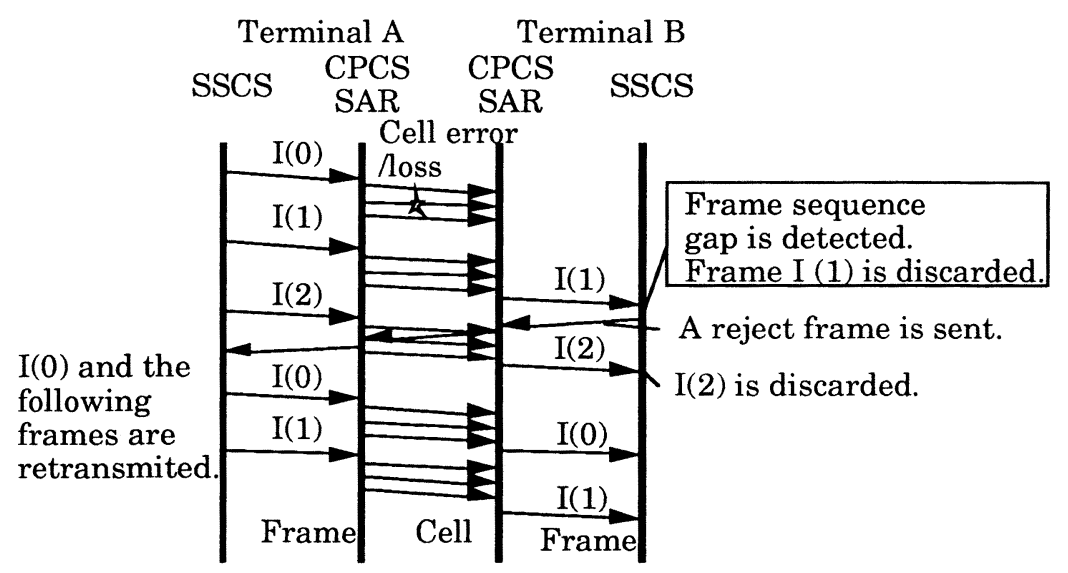

I(n): Frame with a sequence number of $n$.

Figure 5. Sequence chart for GBN error recovery. 


\section{(b) Problem}

With the GBN method, the sender simply retransmits all the data after the error and the receiver simply discards all the data after the error until the error is recovered. Implementation is easy because the sender needs to buffer only a copy of the unacknowledged data and the receiver needs to buffer no more than one frame. Although this method is simple and easy, it leads to unnecessarily retransmitting data that has been correctly received; this may lead to large transmission overhead, especially in environments with a large bit-rate round-trip delay product. B-ISDN has a high bit rate as well as varying round-trip delay. In addition, it uses a small transmission unit, a 53-byte cell, and error or data loss occurs only in this cell. Since GBN error recovery in B-ISDN would retransmit a large number of cells unnecessarily, using it would greatly degrade efficiency.

\subsubsection{Selective-Frame Retransmission}

\section{(a) Procedure}

In the SSCOP and in some options of the TCP, XTP, VMTP, and HDLC, errored data is selectively retransmitted to recover the error. This scheme is called selective-frame retransmission (SFR); it aims for efficient error recovery in environments with moderate to small error rates, moderate to high transmission speed, and varying round-trip delay. As in GBN retransmission, the data units are identified by a sequence number or flag by both the sending and receiving entities. The sequence number may be a pointer based on the amount of data (bytes). As in GBN retransmission, the sender holds a copy of the unacknowledged data in its buffer and the receiver checks if the sequence number of the received data is continuous or not. When an error occurs, the sender retransmits only the errored or lost data.

The retransmission is invoked either by the receiver's notification using a control frame (an S-Frame in HDLC or a USTAT PDU in SSCOP) or by a timeout at the sender. When the receiver receives the retransmitted data, it combines it with the data in the receiving buffer and reorders it. With this scheme, the sender has to have a buffer for the unacknowledged data and the receiver has to have a buffer for the correctly received data. The buffers are larger than those needed for the GBN scheme.

Figure 6 shows a sequence chart for SFR. A cell in frame I(1) is lost and the frame is discarded at the receiving side. When the next frame is received, the receiver detects the sequence gap and requests retransmission of the lost frame by sending a selective reject (SREJ) frame containing the sequence number of the lost frame. All the frames received after the error are contained at the receiver for reordering.

\section{(b) Problem}

The SFR scheme requires a reordering procedure and a larger buffer space at the receiver, which means more overhead than with GBN. However, SFR goes increase transmission efficiency in terms of the total amount of data that needs to be retransmitted. SFR, therefore, reduces the average overall time for sending.

With short frames, the transmission efficiency may not be improved much because of the relatively large overhead for each frame. Large files can be sent 
more efficiently by segmenting them into longer frames. With longer frames, however, the frame loss rate increases for the same cell error/loss rate. The number of correctly received cells will also grow, but they will more likely be retransmitted unnecessarily because they are contained in errored or missing frames.

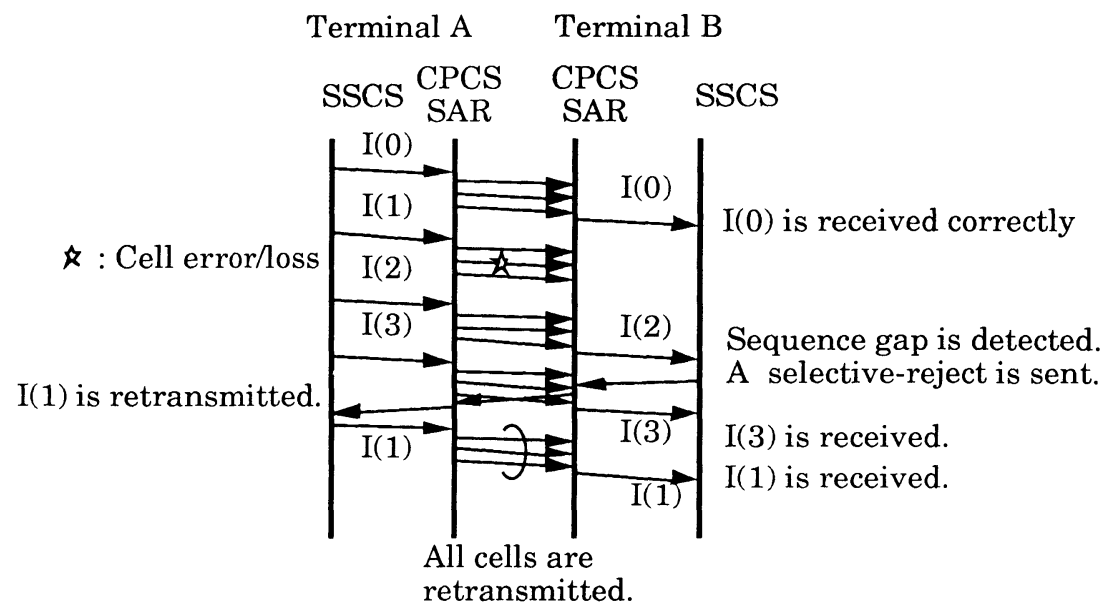

Figure 6. Sequence chart for SFR error recovery.

Although the SFR scheme improves efficiency and total mean response time, as well as total throughput, compared with GBN, its error recovery retransmission performance has to be further improved. This is because in some cases[13], the BISDN cell-loss rate of may be not as small as $10^{-9}$.

\subsubsection{Partial-Frame Retransmission[13][18][19][20]}

The partial-frame retransmission (PFR) method for error recovery, which depends on B-ISDN cell-based transfer, is proposed. It aims to increase transmission efficiency compared to SFR. The basic concept is to retransmit only errored or lost cells; the receiver then combines the cells which were initially received correctly with the retransmitted cells into a complete frame.

\section{(a) Procedure}

With PFR, the frames are given frame-level sequence numbers, as in GBN and SFR. The receiver constructs a frame even if all the constituent cells have not been received correctly. In doing so, the receiver creates a list indicating which cells were received correctly and which were not. The length of the list is minimized by using a bit sequence to indicate each cell's reception result. We call this list a bitmap. To identify the lost or errored cells, an identifier is needed to show each 
cell's location in the frame. AAL-CP Type 3/4 gives a cell-level sequence number (SN) to each cell payload; this SN can be used as an identifier. Although AAL-CP Type 5 does not provide such an identification mechanism, if no VC multiplexing is performed within a user's VP, the VCI value can be used for identification. In other words, the cell's VCI would be incremented each time a new frame is sent.

This bitmap and frame-level sequence number identify the cells to be retransmitted. Although identification by using only the cell-level sequence number would be possible, since this sequence number needs to be long, it would be a greater burden than the proposed identification mechanism.

When a frame is determined to be errored, the receiver notifies the sender of the sequence number of the errored and/or lost frame with its bitmap. The correctly received cells are kept in the receiver buffer until the retransmitted cells are received; the complete frame is then reconstructed and sent to the upper part. When the sender receives an error notification with its cell-level reception result (bitmap), the sender constructs a new frame containing only the requested cells as well as a copy of the bitmap.

When the receiver detects frame losses by gaps in the sequence number, it sends a notification of the losses with the bitmap showing that all the cells were not received. When the receiver receives the retransmitted frame, it reconstructs the complete frame by combining the held cells with the retransmitted cells according to the bitmap contained in the retransmitted frame. These error notifications can be done with control frames (SREJ PDUs in HDLC and USTAT/STAT PDUs in SSCOP). Figure 7 shows a sequence chart for PFR. The second cell of the frame $\mathrm{I}(0)$ is lost; it is requested to be retransmitted by a SREJ with a bit map. The sender sends back a short frame containing the same bitmap and the second cell.

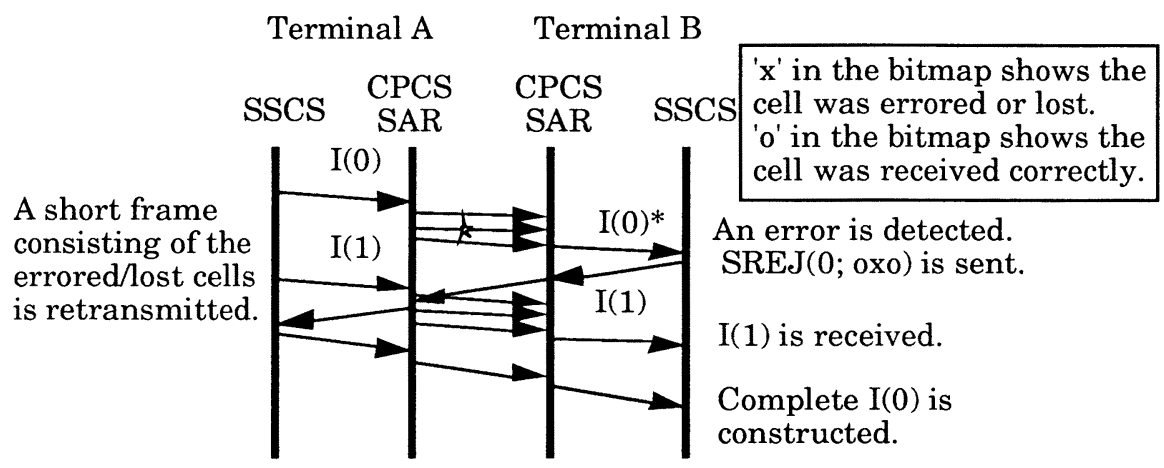

I(0)*: Frame with cell error/loss

Figure 7. Sequence chart for partial frame retransmission.

Though XTP and VMTP provide selective retransmission, their retransmission units are basically user data units (packets etc.) and are much longer than the 
payload length of an ATM cell. In particular, VMTP provides no transmitter procedure for polling the receiver's status and retrying the retransmission if necessary.

\section{(b) Hardware Implementation}

Figures 8 and 9 show the functional blocks needed to provide SFR and PFR. AAL Type $3 / 4$ is assumed. SSCS is assumed to be the SSCOP and the SSCF. The SSCF and higher parts, as well as the ATM and lower parts, are not shown because they are common to the two retransmission schemes. In general, these functional blocks add AAL-SS (frame) overhead and AAL-CP overhead to the data, and segment the data into SAR units with AAL-SAR overhead and an ATM header is added. The data is kept in the transmitter's buffer (Tx buffer) for retransmission. Received cells are processed in reverse order. When the received data is user data, it is sent to its local user. When it is a PDU requesting retransmission, the requested PDUs in the Tx buffer are retransmitted. When the data is polling the receiver status information, the information is sent back in the STAT PDU.

Blocks additional to the ones for SFR are the described below; they are dashed in Fig. 9 and add little overhead.

(1) SD generation based on the received bitmap

This block generates a short SD PDU according to the received bitmap by using the SD PDU in the transmission (Tx) buffer. This function can be performed by the Tx buffer manager; it can also be done with a processor and the proper software.

(2) Creation of bitmap

This function creates a bitmap for every received SD PDU; it can be performed with a register that has the same length as the maximum bitmap length. For example, the approximate number of needed gates would be five [gates/bit] times the bitmap length plus the fixed number of gates.

(3) Check for the initially received SD PDU

This function determines if the received CPCS PDU is an initially received SD PDU or not. If not, the created bitmap is discarded. This function can be performed by a small number of gates.

(4) Receiver partial-frame buffer

This buffer stores the received SD PDUs, even if they are not complete. When it receives a retransmitted partial frame, it reconstructs a complete frame from the stored and the received (partial) frames. This block would be the same size as the SD generation function block.

These additional blocks can be implemented with several thousand gates, using a several-hundred bits bitmap, which corresponds to a maximum frame length of several tens of kilo-bytes. Considering that an AAL Type 5 can be made with several tens of kilogates, this addition is not significant. 

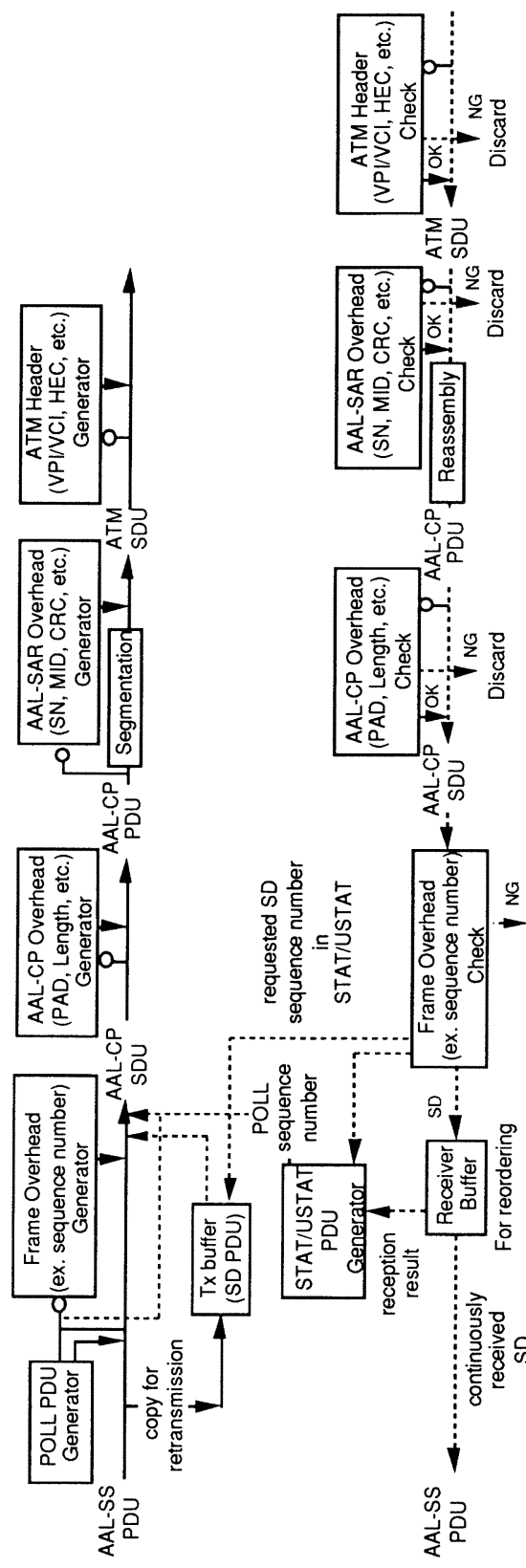

这总
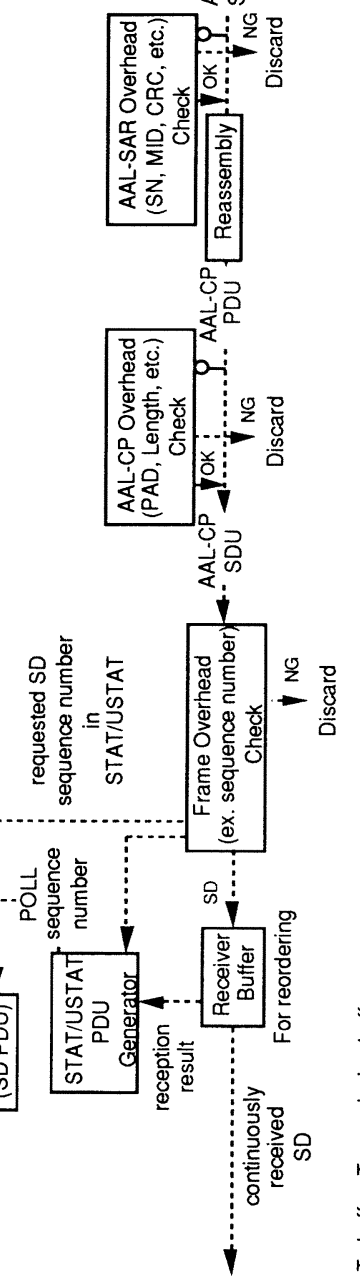

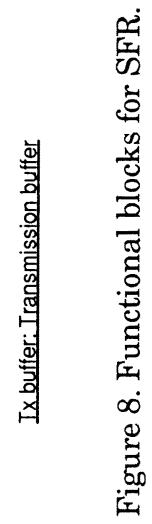




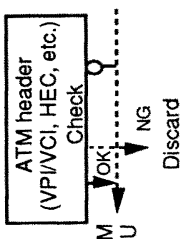

这总
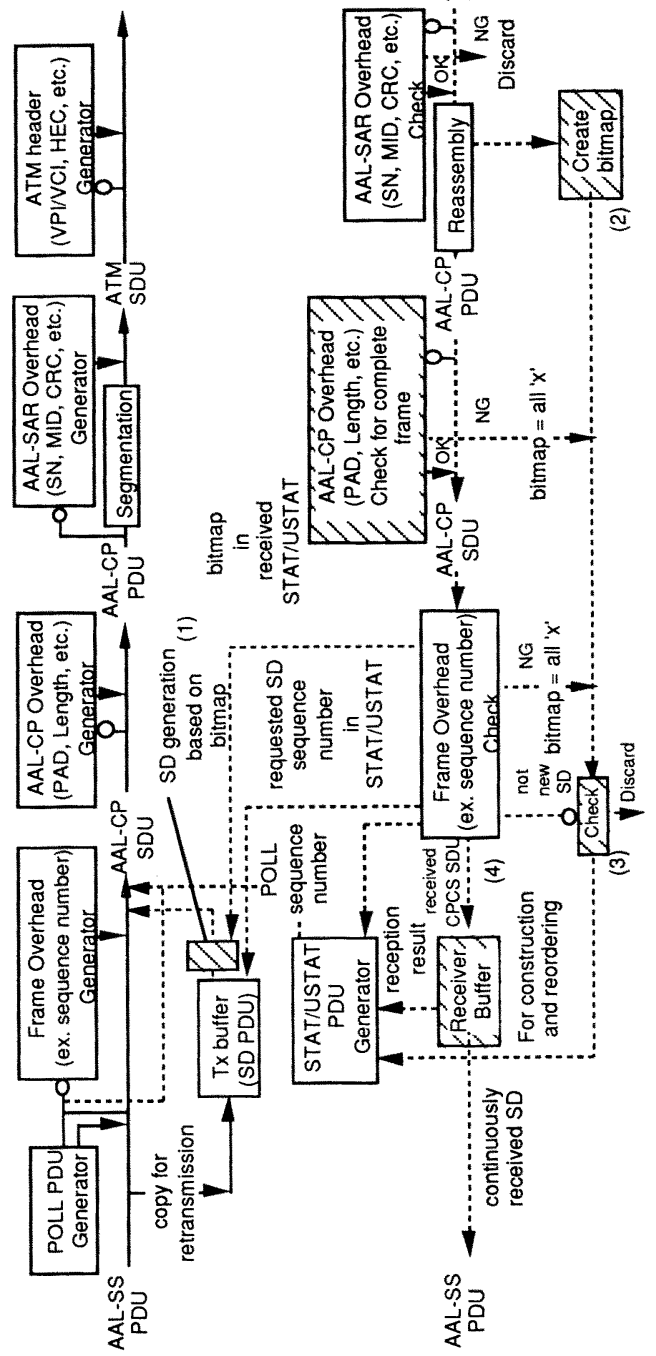

 
PFR can thus be performed with only a little more burden than for GBN or SFR; it would therefore not reduce the processing speed. Additionally, the required receiver buffer space is almost the same size as for SFR if the other conditions are the same. This is because the required buffer space is determined approximately by the product of the link throughput and the delay.

\section{(c) Expected Benefits}

The proposed PFR method will reduce the amount of retransmitted traffic. This will reduce the response time for each frame's acknowledgment and reduce the total traffic volume (initially sent data plus retransmitted data). It will also lower the total cell-loss rate as well as the congestion probability, both of which are the result of the improved efficiency.

\section{Evaluation [18][19][20]}

In this section we evaluate the PFR method in terms of data communication efficiency and total error-rate reduction. These two criteria are coupled with the data communication application requirements for B-ISDN because efficient and low-error-rate communication reduces response time, congestion probability, and charges (when usage-sensitive charging is used).

\subsection{Transmission Efficiency}

Transmission efficiency is defined as:

the amount of correctly transmitted user data divided by the total amount of data for the transmission, including retransmitted data and the header overhead.

To focus on the retransmission scheme's effect on efficiency, the following assumptions are made. The cell-loss rate is assumed to be fixed. The receiver buffer is assumed to be infinite. The cell-loss rate for each cell in the network is independent. The network is modeled as two communicating terminals with a point-to-point link that has a fixed cell-loss rate and a fixed throughput capacity. The sender continuously sends data at a fixed rate.

Following these assumptions, the efficiency can be obtained from the formula below.

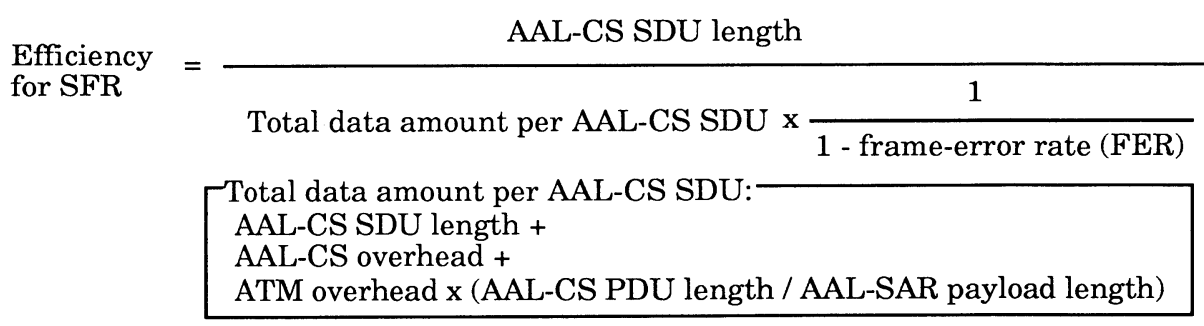


This is because after a frame-error occurs with a probability of FER, another frame is retransmitted ; the total number of frames is thus multiplied by (1+FER + FER x FER + ...).

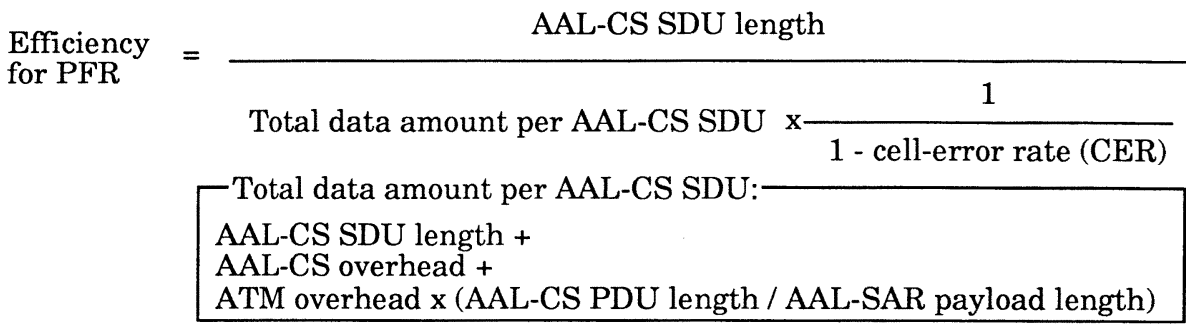

This is because if a cell error occurs, only that cell is retransmitted. If the same cell is again errored, one more cell is retransmitted with a probability of CER. Therefore, the total number of retransmitted cells is multiplied by $(1+$ CER + CER $\mathrm{x}$ CER +...).

In both cases, maximum efficiency is obtained when the error rate is zero.

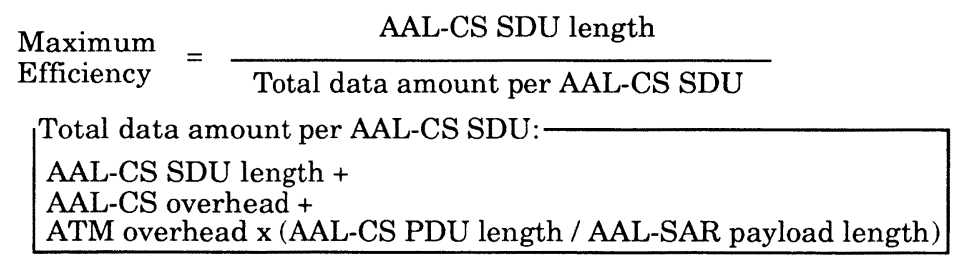

Here,

FER = 1 - (1- CER) $\left[\frac{\text { AAL - CS PDU length }}{\text { AAL - SAR SDU payload length }}\right]$

[\#] is the maximum integer no larger than \#.

AAL-CS overhead : 8 bytes

ATM overhead : 5 bytes

SAR payload : 44 bytes

The evaluation results (Fig. 10) show that the efficiency provided by PFR can be higher than that of SFR for a wide variety of cell-loss rates, for a variety of frame lengths. 


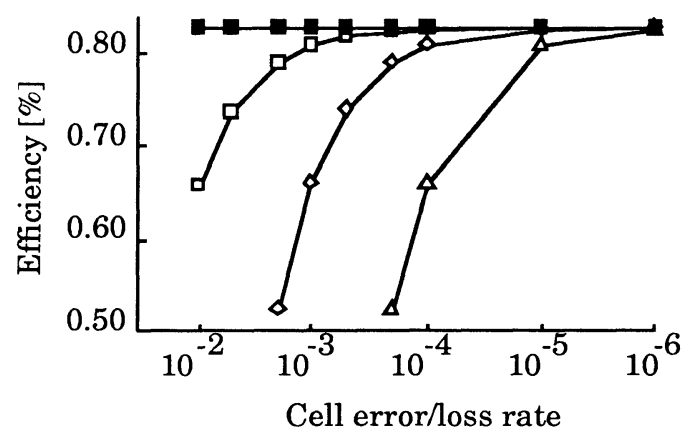

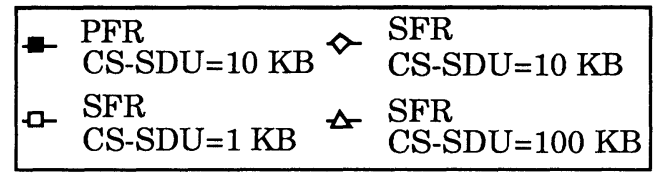

Figure 10. Error recovery efficiency depending on the cell error/loss rate.

\subsection{Total Traffic (Error Rate) Reduction}

To evaluate the transmission efficiency, the cell-loss rate was assumed to be fixed. Actual cell loss, however, depends on the overflow of the finite-size buffers in the nodes, e.g., switches, in the network; it varies depending on the buffer space as well as on the amount traffic of coming into the network. The proposed PFR can reduce the of amount retransmitted traffic compared with GBN and SFR. Therefore, when the terminals try to send a fixed amount of data under the same conditions (link rate, buffer space frame length, etc.), the cell-loss rate may vary depending on the retransmission scheme, i.e., SFR or PFR. In particular, PFR will lower the cellloss rate compared with SFR. In this section we evaluate the total traffic load, including the retransmitted traffic, taking into account the cell overflow at the buffers.

The evaluation was done using computer simulation with the following assumptions:

(1) Several terminals share the output buffer at the egress node of the network; cell loss occurs only at this point (Figure 11).

(2) The data flows in the same direction; there are no resource conflicts with the control information flowing in the reverse direction.

(3) The data frame length is fixed; frames are generated according to a Poisson process.

(4) Cells in a frame are sent to the network at a fixed rate-the maximum rate of the link between the terminal and the network. 
(5) Processing time including propagation delay is assumed to be $5 \mathrm{~ms}$ in any direction.

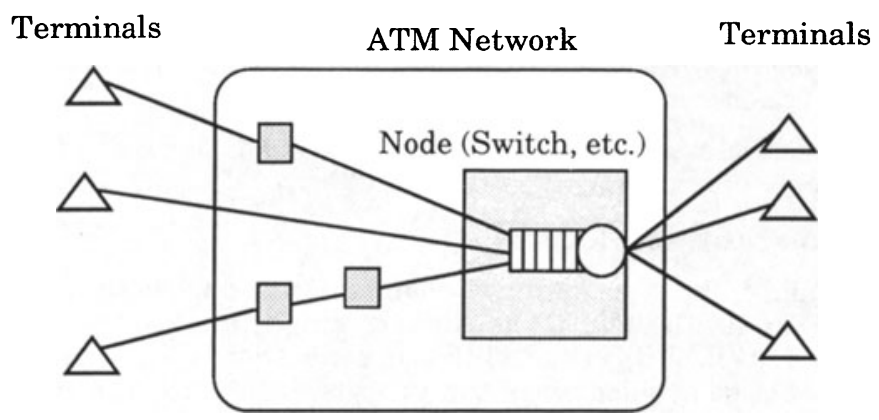

Figure 11. Simulation model for communicating terminals in B-ISDN.

Figure 12 shows the simulation results. The horizontal axis shows the initially generated total traffic load normalized by the output-link rate. The vertical axis shows the cell-loss rate at the output buffer. The frame length and the VC speeds are parameters. The shared-buffer space is assumed to be 127 cells.

Output Speed $=156 \mathrm{Mb} / \mathrm{s}$

Output buffer size $=127$ cell
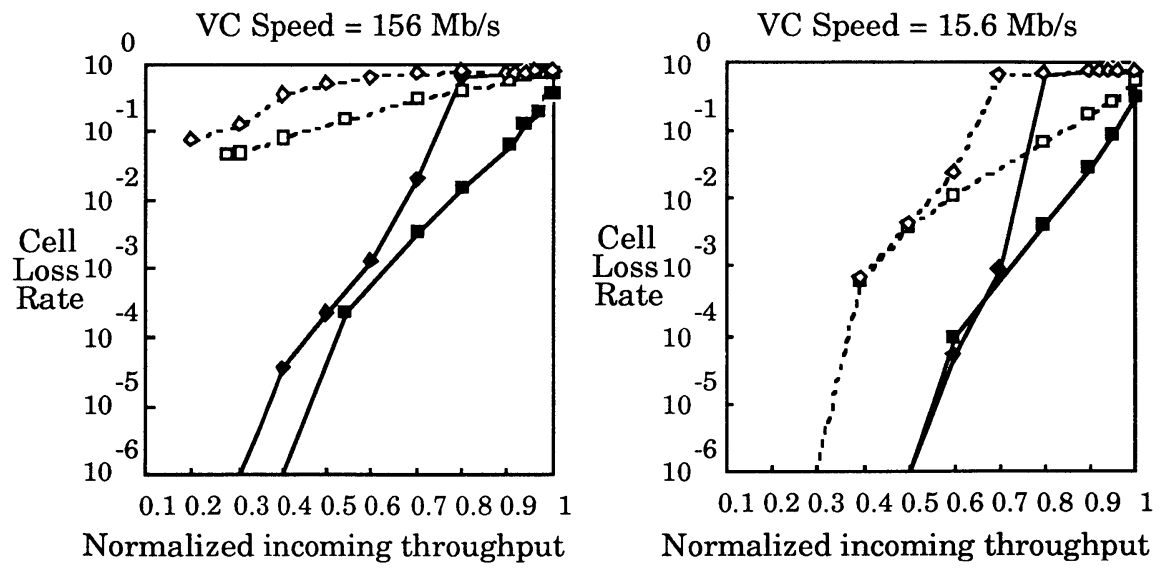

Normalized incoming throughput

Normalized incoming throughput

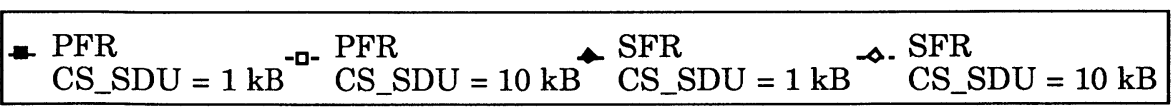

Figure 12. Simulated cell-loss rate depending on the incoming traffic. 
The results show the following:

\section{Dependency on the retransmission scheme}

For all the variations in parameter values, PFR reduces the cell-loss rate by more than $90 \%$ when no congestion occurs. When the frame length is $1 \mathrm{kB}$, SFR generates congestion when the normalized incoming traffic load is 0.8 or greater. PFR keeps the cell-loss rate below 0.01 .

Therefore, the cell-loss rate can be greatly reduced and congestion can be avoided for a wide variety of parameter values by using PFR.

\section{Dependency on the frame length}

Even if PFR is used, the network may become congested when the frame length is $10 \mathrm{kB}$, the $\mathrm{VC}$ rate is $156 \mathrm{Mbps}$, and the incoming traffic load is more than 0.2 . This is also the case if SFR is used. To avoid congestion, the use of frames longer than $10 \mathrm{kB}$ should be avoided when the VC rate is $156 \mathrm{Mbps}$ or greater and the output buffer space is less than 127 cells. For frames that are $1 \mathrm{kB}$ or less, a relatively low cell-loss rate can be provided for traffic load lighter than 0.6.

\section{Dependency on the VC rate}

The cell-loss rate can be reduced by reducing the VC rate. For a given cell-loss rate (for example 0.001), more traffic can enter the network with a lower VC rate. For SFR, the VC rate at which the network starts to become congested is independent of the incoming traffic load.

\section{Conclusion}

This paper described the functions needed for data communication in B-ISDN and classified the error recovery methods that can be used by the end-to-end protocol for data communication. The partial-frame retransmission (PFR) scheme was proposed; its performance was compared with that of selective-frame retransmission (SFR) from the viewpoints of implementation complexity, efficiency, total traffic load, and cell-loss probability. Compared to SFR, only a few additional function blocks are required for PFR.

The evaluation results showed that PFR can provide higher efficiency for a wide variety of parameter values compared with SFR. They also showed that PFR reduced the cell-loss rate to less than 0.1 . In particular, when the incoming traffic load is relatively high, PFR can avoid the congestion that SFR allows.

More precise evaluations are needed that take into account the variations in incoming traffic load and such parameters as propagation delay. The proposed PFR with flow control based on cell-level transmission should be considered for use in the new SSCS protocol for data communication in B-ISDN.

\section{Acknowledgments}

We wish to thank Mr. Takeo Koinuma, Mr. Tatsuro Takahashi, and Mr. Hirokazu Ohnishi for giving us the chance to research this topic. We also wish to thank Dr. 
Kou Miyake, Mr. Hiroyuki Ichikawa, Mr. Toshikazu Suzuki, and Mr. Michiharu Mito for their fruitful discussions.

\section{References}

[1] ITU-T Draft Recommendation M.3010, "Principles for a telecommunications management network."

[2] ITU-T Recommendation I.150, "Integrated services digital network (ISDN) general structure and service capabilities," 1991.

[3] ITU-T Recommendation I.361, "B-ISDN ATM layer specification," 1992.

[4] ITU-T Recommendation I.362, "B-ISDN ATM adaptation layer (AAL) functional description," 1992.

[5] ITU-T Recommendation I.363, "B-ISDN ATM adaptation layer (AAL) specification," 1992.

[6] ITU-T Recommendation Q.2110, "B-ISDN signalling ATM adaptation layer service specific connection oriented protocol (SSCOP)," 1994.

[7] J. Heinanen, "Multiprotocol Encapsulation over ATM Adaptation Layer 5," IETF RFC 1483, July 1993.

[8] J. Postel, "Transmission Control Protocol," IETF RFC 793, September 1981.

[9] J. Postel, "Internet Protocol," IETF RFC 791, September 1981.

[10] Protocol Engines; “XTP Protocol Definition,” Rev. 3.6, January 1992.

[11] D. Cheriton, "VMTP: Versatile Message Transaction Protocol protocol specification," IETF RFC 1045, February 1988.

[12] ISO I IEC 4335; "Consolidation of Elements of Procedures (HDLC)."

[13] I. Inoue, S. Chaki, and N. Morita, "ATM Techniques for High Speed Data Communication," International Networking Conference 92 Proceedings, pp. 377-386, June 1992.

[14] ITU-T Recommendation Q.703, "Specifications of signalling system No. 7 signalling link," 1988.

[15] ITU-T Recommendation X.25, "Interface between data terminal equipment (DTE) and data circuit-terminating equipment (DCE) for terminals operating in the packet mode and connected to public data networks by dedicated circuit," 1992.

[16] ITU-T Recommendation Q.921, "ISDN user-network interface - data link layer specification," 1988.

[17] ITU-T Recommendation Q.922, "ISDN data link layer specification for frame mode bearer services," 1992.

[18] NTT, "Proposed SSCS Specification for AAL Type 3 Protocol," ITU-T contribution, COM XVIII, D.1848, December 1991. 
[19] I. Inoue, N. Morita, and H. Ohnishi, "Proposal and Evaluation of an AAL Protocol with Error Recovery by Cell by Cell Retransmission," IEICE Autumn National Conference, B-296, September 1991 (in Japanese).

[20] I. Inoue, N. Morita, and H. Ohnishi, "Evaluation of an AAL Protocol Having an Error Recovery Function based on Retransmitting Lost Cells," IEICE Technical Report 91, SSE91-94, November 1992 (in Japanese). 